\title{
Wikabularies and the Like - Community-Based Knowledge Resources on the Web
}

\author{
Iryna Gurevych \\ UKP Lab, Computer Science Department \\ Technical University of Darmstadt, Germany
}

In recent years, community-based knowledge resources on the Web have gained increasing popularity. Resources, such as the community-based encyclopedia Wikipedia or the community-based dictionary Wiktionary, have been employed as background knowledge sources in processing unstructured textual information in multiple languages. They yielded excellent results comparable to or better than the manually constructed resources by experts.

In the talk, we will discuss the major properties of already established and emerging community-based knowledge resources, whose most prominent examples are Wikipedia http://www.wikipedia.org and its dictionary spin-offs Wiktionary http://www.wiktionary.org and OmegaWiki http://www.omegawiki.org. We will analyze how they represent word senses and synonyms, which are fundamental units of knowledge about the words for processing human languages. Finally, we will conclude by outlining some promising application areas and future research directions.

We expect that the results of this research will have clear impact beyond natural language processing. They can also inspire related fields concerned with unstructured information management, knowledge representation and data integration. 